\title{
Factors associated with non-response to lactulose therapy in cirrhotic patients with minimal hepatic encephalopathy
}

\author{
Shireen Ahmed, Md. Golam Azam, Indrajit Kumar Datta, Md. Nazmul Hoque, Tareq M Bhuiyan \\ Department of Gastroenterology Hepatobiliary and Pancreatic Disorders, \\ BIRDEM General Hospital, Dhaka, Bangladesh
}

\begin{abstract}
Background and objectives: Minimal hepatic encephalopathy (MHE) impairs health related quality of life and predicts overt hepatic encephalopathy (HE) in cirrhotic patients. Lactulose is effective in the treatment of MHE. But the response to lactulose treatment depends on several factors. This study was aimed to find out the contributing factors to non-response to lactulose therapy.

Materials and methods: The study was carried out at the BIRDEM general hospital from September, 2013 to March, 2015. Sixty patients were enrolled to assess the response of lactulose therapy in cirrhotic patients with MHE. MHE was diagnosed based on abnormal psychometric tests namely, number connection test (NCT), digit symbol test (DST) and high serum ammonia level. A daily dose of 30-60 ml of lactulose was given to all patients for one month. The response to treatment with regard to MHE was determined after one month using defined criteria. The response was graded as responder and non-responder.

Results: The mean age of the study population was $57.0 \pm 10.3$ years. Out of 60 cases, 46 (77\%) were male and $39(65 \%)$ had diabetes. Out of 60 enrolled MHE cases, $16(27 \%)$ had Child-Turcotte-Pugh-A (CTP-A) score and 44 (73\%) belonged to CTP-B \& C category.

Out of 60 MHE cases, 23 (38.3\%) showed improvement in their MHE status based on normalization of psychometric tests and reduction of serum ammonia level to $\leq 32 \mu \mathrm{mol} / \mathrm{L}$. Age, gender and diabetes were not associated with the response to lactulose therapy. Low baseline arterial pressure was significantly $(p=0.003)$ associated with non-response to lactulose treatment. The mean baseline ammonia level was higher significantly among the nonresponders compared to the responders $(83.6 \pm 21.4 \mu \mathrm{mol} / \mathrm{L}$ vs $58.8 \pm 19.8 \mu \mathrm{mol} / \mathrm{L}, \mathrm{p}<0.001)$. Compared to responders, low serum sodium and potassium and raised serum bilirubin levels of non-responders at baseline were found significantly $(\mathrm{p}<0.05)$ associated with non-response to one month of lactulose treatment. Initial hemoglobulin, peripheral leucocyte and platelet counts did not have any effect on the response to lactulose treatment in MHE cases.

Conclusions: The status of MHE in patients with cirrhosis improved by one-month treatment with lactulose. Baseline low arterial pressure, hyperammonemia, hypokalemia and hyponatremia were major contributors to non-response to lactulose therapy. The findings of the study would be useful in treating patients of cirrhosis with MHE.
\end{abstract}

IMC J Med Sci 2018; 12(1): 15-21

\section{Introduction}

Hepatic encephalopathy (HE) is defined as a spectrum of neuropsychiatric abnormalities in patients with liver dysfunction, after exclusion of other known brain disease. Hepatic encephalopathy is characterized by personality changes, intellectual

Address for Correspondence:

Dr. Shireen Ahmed, Registrar, Department of Gastroenterology Hepatobiliary and Pancreatic Disorders, BIRDEM General Hospital, 122 Kazi Nazrul Islam Avenue, Dhaka, Bangladesh.E-mail: a.alwasi15@gmail.com 
impairment, and a depressed level of consciousness. An important prerequisite for the syndrome is diversion of portal blood into the systemic circulation through porto-systemic collateral vessels [1]. The development of hepatic encephalopathy negatively impacts patient survival. The survival probability of patients with $\mathrm{HE}$ requiring hospitalization has been reported to be $42 \%$ at 1 year of follow-up and $23 \%$ at 3 years. Approximately, $30 \%$ of patients dying of end-stage liver disease experience significant encephalopathy [2]. The economic burden of hepatic encephalopathy is substantial. After ascites, hepatic encephalopathy is the second most common reason for hospitalization of cirrhotic patients in the United States [3]. Apart from HE, a considerable number of patients with cirrhosis also develop minimum hepatic encephalopathy (MHE). MHEis a condition in patients with cirrhosis of the liver, who has normal mental and neurological status on standard clinical examination while exhibit a number of neuropsychiatric and neurophysiological defects [4]. The prevalence of MHE varies from 30 to $70 \%$ in cirrhotic patients without overt hepatic encephalopathy $[5,6,7]$. MHE is associated with increased progression to $\mathrm{HE}$ and diminished quality of life [6,7]. Hence, psychometric tests are recommended to screen patients with cirrhosis for detecting $\mathrm{MHE}$.

The diagnostic criteria for MHE rest on careful patient history, physical examination, normal mental status examination, demonstration of abnormalities in cognition and/or neurophysiological function, and exclusion of concomitant neurological disorders [8]. MHE is considered clinically relevant for at least three reasons. It impairs patient's daily functioning and health-related quality of life $[9,10]$. Second, it predicts the development of overt HE. Finally, it is associated with a poor prognosis [11]. In a variety of psychometric tests listed in the medical literature, DST and NCT part A were reported to have the advantages of simplicity and reliability [4].

Gut-derived nitrogenous substances are universally acknowledged to play a major role in the pathogenesis of hepatic encephalopathy. Pathogenesis of MHE is thought to be similar to that of overt HE. Specifically, ammonia is thought to be a critical factor in the pathogenesis [12].
Altered glio-neuronal communication as a result of low grade astrocyte swelling found in $\mathrm{HE}$, have been noticed in patients with MHE and is the basis of new diagnostic tests for detecting MHE like critical flicker frequency and magnetic resonance spectroscopy [13].

Presently, lactulose is the mainstay of treatment for MHE [14,15]. Lactulose is metabolized into lactic and acetic acids, which results in acidification of the gastrointestinal lumen. Gastrointestinal acidification ultimately inhibits the production of ammonia by coliform bacteria. Lactulose also acts as a cathartic. The typical dosage of lactulose is 30 ml 2-4 times per day, adjusted to achieve two to four soft stools/day [16]. Lactulose has been shown to improve cognitive function and health-related quality of life in patients with MHE [9]. A recent study has attributed lack of response to lactulose treatment in cirrhotic patients with MHE to low serum sodium levels and high serum ammonia levels [17]. However, not all patients with MHE respond to lactulose and the efficacy of lactulose varies from 40 to $70 \%$ in various studies [17]. In view of the above, the present study was aimed to evaluate the contributing factors of non-response to lactulose treatment in cirrhotic patients with MHE.

\section{Methodology}

This prospective, longitudinal study was conducted to find the contributors to non-response to lactulose therapy in patients of MHE with cirrhosis of liver at the Department of Gastroenterology Hepatobiliary and Pancreatic Disorders, BIRDEM General Hospital, Dhaka, Bangladesh from September, 2013 to March 2015. The study was approved by the Ethical Review Committee (ERC) of Diabetic Association of Bangladesh (BADAS). Cases were selected by purposive sampling technique and informed written consent was obtained.

Patients of cirrhosis of liver, aged $>18$ years, having MHE with normal mental status with no history of taking lactulose and any antibiotics in the past 12 weeks were included in this study. Normal mental status was assessed by mini mental state examination (MMSE score $\geq 24$ ) [18] and MHE was diagnosed by abnormal psychometric tests (NCT-B >30 second and DST >90 second 
including the error correction time) and raised serum ammonia ( $\geq 32 \mu \mathrm{mol} / \mathrm{L})$ [4]. Cirrhosis was diagnosed based on clinical features, laboratory parameters, abdominal ultrasonography and endoscopic findings.

The mini-mental state examination (MMSE) is a brief 30-point questionnaire test used to screen for cognitive impairment. It is also used to estimate the severity of cognitive impairment and to follow the course of cognitive changes in an individual over time, thus making it an effective way to document an individual's response to treatment. If the score is found $\geq 24$ then it denotes that the person has normal mental status [18]. NCT-B is a test of visuo-spatial orientation and psychomotor speed. The subject is shown a sheet of paper with 25 numbered circles which are randomly spread over the paper. The task is to connect the circles from 1-25 within 30 seconds. Test result is the time needed by the subject including error correction time [19]. In DST, the subject is given a series of double-boxes with a number given in the upper part. The task is to draw a symbol pertinent to this number into the lower part of the boxes. Nine fixed pairs of numbers and symbols are given at the top of the test sheet. Test result is the number of boxes correctly filled within 90 seconds. Pathological test results indicate a deficit in visuoconstructive abilities [20].

Cirrhotic patients who had HE or a history of HE within last three month, history of taking lactulose, any antibiotics, antidepressants, sedatives, prokinetic and antispasmodic drugs, alcohol intake, gastrointestinal hemorrhage or spontaneous bacterial peritonitis during the past 12 weeks, previous transjugular intrahepatic portosystemic shunt or shunt surgery, significant co-morbid illness such as heart, respiratory or renal failure, neurological diseases such as Alzheimer's disease, Parkinson's disease, non-hepatic metabolic encephalopathy, colour blindness, mature cataract, diabetic retinopathy, were excluded from the study.

Lactulose was given to all patients with the dose of 30-60 ml daily for one month and ensured that stool passed 2-3 times per day. Patients on diuretics and beta blocker for ascites or edema and esophageal varix continued the maintenance dose. All patients receiving lactulose were followed up after one month and assessed for the response of lactulose. Response to lactulose on the status of MHE was determined after one month by NCT-B, DST and serum ammonia level. Response was graded as responders and non-responders to lactulose therapy. Responder was defined as cases with normalization of psychometric tests and reduction of serum ammonia level to $\leq 32 \mu \mathrm{mol} / \mathrm{L}$, while non-responder was cases with abnormal psychometric tests and no reduction or increased serum ammonia. Responder and non-responder groups were compared to find the contributing factors to non-response to lactulose treatment. Also, Child-Turcotte-Pugh (CTP) and Model for End Stage Liver Disease (MELD) scorings were used to compare the prognostic status of responder and non-responder to lactulose therapy [21]. CTP score was determined as described in Table- 1 and MELD score was calculated by the formula: [3.8\{ in S. bilirubin $(\mathrm{mg} / \mathrm{dl})\}+11.20$ (in INR) +9.3 in serum creatinine $(\mathrm{mg} / \mathrm{dl})\}+6.4$ ]. Data was analyzed by chi-square and student's t-tests, where applicable.

\section{Results}

The study aimed to assess the response to lactulose therapy in patients of cirrhosis of liver with MHE. Total 60 patients were enrolled in the study. The mean age of participants was $57.0 \pm 10.3$ years. Out of 60 cases, 46 (77\%) were male and 39 $(65 \%)$ had diabetes. Hepatitis B virus was positive in $13(22 \%)$ cases while $9(15 \%)$ had hepatitis C virus. Out of 60 enrolled cases, $16(27 \%)$ had CTP-A score and 44 (73\%) belonged to CTP-B \& $\mathrm{C}$ category (Table-2).

Of the 60 MHE cases, 37 (61.7\%) were nonresponders to one-month lactulose treatment while remaining $23(38.3 \%)$ were responders based on normalization of psychometric tests and reduction of serum ammonia level to $\leq 32 \mu \mathrm{mol} / \mathrm{L}$. Age, gender and diabetes were not associated with the response to lactulose therapy (Table-2). The mean baseline arterial pressure was significantly $(\mathrm{p}=0.003)$ low in non-responders $(76.0 \pm 10.6$ $\mathrm{mmHg})$ than that of responders $(84.5 \pm 9.9$ $\mathrm{mmHg}$ ). The mean baseline ammonia level was higher significantly among the non-responders compared to the responders $(83.6 \pm 21.4 \mu \mathrm{mol} / \mathrm{L}$ vs $58.8 \pm 19.8 \mu \mathrm{mol} / \mathrm{L}, \mathrm{p}<0.001)$. The mean serum 
ammonia level reduced significantly among the responders $(21.2 \pm 5.5 \mu \mathrm{mol} / \mathrm{L})$ after one month of lactulose therapy while it remained high in non- responders $\quad(88.8 \pm 17.7 \quad \mu \mathrm{mol} / \mathrm{L}) . \quad$ The level remained about four times higher in nonresponders on follow up after one month.

Table-1: Child-Turcotte-Pugh score

\begin{tabular}{llll}
\hline \multicolumn{1}{c}{ Score } & \multicolumn{1}{c}{$\mathbf{1}$} & \multicolumn{1}{c}{$\mathbf{2}$} & \multicolumn{1}{c}{$\mathbf{3}$} \\
\hline Encephalopathy & Absent & Mild (grade I-II) & Severe(grade III-IV) \\
Bilirubin(mg/dl) & $<2.0$ & $2.0-3.0$ & $>3$ \\
Albumin (gm/L) & $>3.5$ & $3.5-2.8$ & $<2.8$ \\
Prothrombin time (seconds) & $<4$ & $4-6$ & $>6$ \\
Ascites & Absent & Mild- moderate & Moderate-severe
\end{tabular}

Note: Score 5-6= Child class A; Score 7-9= Child class B; Score $>10=$ Child class $C$.

Table-2: Baseline demographic, clinical and biochemical parameters contributing response to lactulose treatment in MHE patients $(n=60)$

\begin{tabular}{|c|c|c|c|}
\hline \multirow[b]{2}{*}{ Parameter } & \multicolumn{2}{|c|}{ Base line status of } & \multirow[b]{2}{*}{ p value } \\
\hline & $\begin{array}{c}\text { Responder } \\
(\mathbf{n}=23)\end{array}$ & $\begin{array}{c}\text { Non-responder } \\
(\mathbf{n}=\mathbf{3 7})\end{array}$ & \\
\hline Age in years (Mean \pm SD) & $58.3 \pm 10.7$ & $56.7 \pm 9.9$ & 0.61 \\
\hline \multicolumn{4}{|l|}{ Gender $(\mathrm{n}, \%)$} \\
\hline Male $(n=46)$ & $18(39.1)$ & $28(60.9)$ & 0.81 \\
\hline Female $(n=14)$ & $5(35.7)$ & $9(64.3)$ & \\
\hline \multicolumn{4}{|l|}{ DM status $\mathrm{n}(\%)$} \\
\hline $\mathrm{DM}(\mathrm{n}=39)$ & $13(56.5)$ & $26(70.3)$ & 0.277 \\
\hline $\operatorname{NDM}(n=21)$ & $10(43.5)$ & $11(29.7)$ & \\
\hline \multicolumn{4}{|l|}{ CTP score n $(\%)$} \\
\hline CTP-A $(n=16)$ & $13(81.3)$ & $3(18.7)$ & $<0.001$ \\
\hline CTP-B \& C $(n=44)$ & $10(22.7)$ & $34(77.3)$ & \\
\hline MELD & $38.1 \pm 13.5$ & $44.9 \pm 13.7$ & 0.064 \\
\hline MAP mmHg $($ Mean \pm SD $)$ & $84.5 \pm 9.9$ & $76.0 \pm 10.6$ & 0.003 \\
\hline S. Ammonia $(\mu \mathrm{mol} / \mathrm{L})$ & $58.8 \pm 19.8$ & $83.6 \pm 21.4$ & 0.001 \\
\hline S. Sodium (mmol/L) & $140.3 \pm 3.9$ & $127.4 \pm 6.0$ & 0.001 \\
\hline S. Potassium (mmol/L) & $4.2 \pm 0.6$ & $3.8 \pm 0.7$ & 0.021 \\
\hline S. Bilirubin (mg/dl) & $1.3 \pm 0.8$ & $2.7 \pm 2.8$ & 0.030 \\
\hline S. Total protein $(\mathrm{mg} / \mathrm{dl})$ & $66.0 \pm 9.8$ & $63.9 \pm 10.4$ & 0.440 \\
\hline S. Albumin (mg/dl) & $26.3 \pm 7.7$ & $24.7 \pm 4.4$ & 0.256 \\
\hline S. Creatinine (mg/dl) & $1.4 \pm 1.2$ & $1.3 \pm 0.4$ & 0.610 \\
\hline S. ALT (U/L) & $49.8 \pm 22.9$ & $47.9 \pm 30.9$ & 0.794 \\
\hline $\mathrm{RBS}(\mathrm{mmol} / \mathrm{L})$ & $10.36 \pm 4.56$ & $10.22 \pm 5.14$ & 0.916 \\
\hline $\mathrm{Hb} \mathrm{gm} / \mathrm{dl}$ & $9.7 \pm 1.6$ & $9.9 \pm 1.4$ & 0.708 \\
\hline Total WBC $\left(10^{3} / \mathrm{uL}\right)$ & $5.8 \pm 3.0 .4$ & $7.2 \pm 3.2$ & 0.96 \\
\hline Platelet $\left(10^{3} / \mathrm{uL}\right)$ & $120.0 \pm 62.7$ & $169.5 \pm 38.3$ & 0.54 \\
\hline
\end{tabular}

Note: ${ }^{a}$ Serum ammonia levels after one month of lactulose therapy were $21.2 \pm 5.5 \mu \mathrm{mol} / \mathrm{L}$ and $88.8 \pm 17.7$ in responders and non-responders respectively. DM - diabetes mellitus; NDM - Non diabetes mellitus; MAP - mean arterial pressure; CTP - Child-Turcotte-Pugh; MELD - Model for end stage liver disease; RBS - Random blood sugar; WBC-white blood cell. $p$ value was calculated using $t / \lambda^{2}$ tests (where applicable). 
Compared to responders, low serum sodium and potassium and raised serum bilirubin levels of nonresponders at baseline were found significantly $(p<0.05)$ associated with non-response to one month of lactulose treatment (Table-2). Initial hemoglobulin, leucocyte and platelets as well as other biochemical parameters mentioned in Table-2 did not have any effect on the response to lactulose treatment in MHE cases.

\section{Discussion}

MHE is a well-recognized cause of diminished quality of life and predisposes overt HE. Oral lactulose therapy is a treatment option for MHE. But some patients do not respond to lactulose. This prospective study was carried out to assess the response of lactulose therapy in patients with cirrhosis and MHE. In this study, sixty patients having cirrhosis of liver and MHE were included. This study showed more than half of the patients were non-responder to lactulose $37(61.7 \%)$. It was assumed that non-responders could be less if patients could be followed up for longer duration with lactulose therapy. MHE reversed in $64.5 \%$ patients when treated with lactulose for three months [9].

In this study, it was found that the higher CTP score and low mean arterial pressure (MAP) as contributing factors to non-response to lactulose. As reported earlier, the present study showed that advance liver disease had less chance to response to lactulose therapy [22]. Splanchnic and systemic arterial vasodilatation is a hallmark of the progression of portal hypertension in patients with cirrhosis and leads to decreased effective circulating blood volume and ultimately to a decrease in blood pressure as well as MAP. This process is mediated by a number of endogenous substances, including nitric oxide (NO), carbon monoxide (CO), glucagon, prostacyclin, adrenomedullin, and endogenous opiates that are released or act locally in the vasculature in response to mechanical and inflammatory signals $[23,24]$.

Hyperammonemia, hyponatremia, hypokalemia as well as increased bilirubin were found as predictors of non-response to lactulose treatment. Different studies also demonstrated that hyperammonemia, serum sodium concentration $<135 \mathrm{mmol} / \mathrm{L}$ and serum potassium level of $<4 \mathrm{mmol} / \mathrm{L}$ were associated with greater frequency of hepatic encephalopathy as well as minimal hepatic encephalopathy and reduced response to lactulose therapy $[17,25,26]$. Different factors are responsible for development of hepatic encephalopathy. These factors include the production of neurotoxins, altered permeability of the blood brain barrier, and abnormal neurotransmission. Ammonia is the best-described neurotoxin involved in HE. It is produced primarily in the colon, where bacteria metabolize proteins and other nitrogen-based products into ammonia. Enterocytes synthesize ammonia from glutamine $[27,28,29]$. Once produced, ammonia enters the portal circulation and, under normal conditions, is metabolized and cleared by hepatocytes. In cirrhosis and portal hypertension, reduced hepatocyte function and portosystemic shunting contribute to increased circulating ammonia levels. Acute hyperammonemia has direct effect on brain edema, astrocyte swelling, and the transport of neurally active compounds, thus contributing to $\mathrm{HE}[30,31,32]$. Hyponatremia and hypokalemia are risk factor for hepatic encephalopathy as well as MHE, so if the levels are more reduced then the response to lactulose therapy is also decreased $[25,26]$.

High baseline total leukocyte counts and creatinine level, and low platelet count have been reported to associated with nonresponse to lactulose [33]. However, this study failed to find any significant influence of these parameters with non-response to lactulose therapy (Table 2). This different outcome might be due to small sample size and different patient selection criteria.

The present study evaluated the contributing factors to non-response to oral lactulose therapy in cirrhotic patients with MHE. On analysis, baseline low MAP, high CTP score, low serum sodium and potassium levels, increased serum bilirubin and hyperammonemia were found to be associated with non-response to lactulose therapy.

\section{Author's contributions}

SA was involved in study design, data collection, literature review, data analysis and manuscript writing. MGA was involved in study design, 
statistical analysis and manuscript writing. IKD and $\mathrm{MNH}$ was involved in literature review and manuscript writing. TMB was involved in editing and final approval of the manuscript.

\section{Competing interest}

Authors declare no conflict of interest.

\section{Funding}

None

\section{References}

1. Ferenci P, Lockwood A, Mullen K, Tarter R, Weissenborn K, Blei AT. Hepatic encephalopathy- definition, nomenclature and quantification: final report of the working party at the 11th World Congresses of Gastroenterology, Vienna, 1998. Hepatology. 2002; 35(3): 716-721.

2. Das A, Dhiman RK, Saraswat VA, Verma M, Naik SR. Prevalence and natural history of subclinical hepatic encephalopathy in cirrhosis. J Gastroenterol Hepatol. 2001; 16(5): 531-535.

3. Groeneweg M, Moerland W, Quero JC, Hop WC, Krabbe PF, Schalm SW. Screening of subclinical hepatic encephalopathy. J Hepatol. 2000; 32(5): 748-753.

4. Saxena N, Bhatia M, Joshi YK, Garg PK, Dwivedi SN, Tandon RK. Electrophysiological and neuropsychological tests for the diagnosis of subclinical hepatic encephalopathy and prediction of overt encephalopathy. Liver Int. 2002; 22(3): 190-197.

5. Groeneweg M, Quero JC, De Bruijn I, Hartmann I, Essink-bot MI, Hop WCJ, et al. Subclinical hepatic encephalopathy impairs daily functioning. Hepatology. 1998; 28(1): 45-49.

6. Marchesini G, Bianchi G, Amodio P, Salerno F, Merli M, Panella C, et al. Italian Study Group for Quality of Life in Cirrhosis. Factors associated with poor health-related quality of life of patients with cirrhosis. Gastroenterology. 2001; 120(1): 170-178.

7. Schomerus H, Hamster W. Quality of life in cirrhotics with minimal hepatic encephalopathy. Metab Brain Dis. 2001; 16(1): 37-41.
8. Amodio P, Del Piccolo F, Marchetti P, Angeli $\mathrm{P}$, Iemmolo $\mathrm{R}$, Caregaro $\mathrm{L}$, et al. Clinical features and survival of cirrhotic patients with subclinical cognitive alterations detected by the number connection test and computerized psychometric tests. Hepatology. 1999; 29(6): 1662-1667.

9. Prasad S, Dhiman RK, Duseja A, Chawla YK, Sharma A, Agarwal R. Lactulose improves cognitive function and health-related quality of life in cirrhotic patients with minimal hepatic encephalopathy. Hepatology. 2007; 45(3): 549-559.

10. Watanabe A, Tuchida T, Yata Y, Kuwabara Ye. Evaluation of neuropsychological function in patients with liver cirrhosis with special reference to their driving ability. Metab Brain Dis. 1995; 10(3): 239-248.

11. Cash WJ, Mc Conville P, McDermott E, McCormick PA, Callender ME, McDougall NI. Current concepts in the assessment and treatment of hepatic encephalopathy. $Q \mathrm{~J} \mathrm{Med}$. 2010; 103(1): 9-16.

12. Lockwood AH, Yap EW, Rhoades HM, Wong WH. Altered cerebral blood flow and glucose metabolism in patients with liver disease and minimal encephalopathy. J Cerebr Blood F Met. 1991; 11: 331-36.

13. Sharma P, Sharma BC, Puri V, Sarin SK. Critical flicker frequency: diagnostic tool for minimal hepatic encephalopathy. J Hepatol. 2007; 47(1): 67-73.

14. Dhiman RK, Sawhney MS, Chawla YK, Das G, Ram S, Dilawari JB. Efficacy of lactulose in cirrhotic patients with subclinical hepatic encephalopathy. Dig Dis Sci. 2000; 45(8): 1549-52.

15. Watanabe A, Sakai T, Sato S, Imai F, Ohto M, Arakawa Y, et al. Clinical efficacy of lactulose in cirrhotic patients with and without subclinical hepatic encephalopathy. Hepatology. 1997; 26(6): 1410-14.

16. Bajaj JS, Sanyal AJ, Bell D, Gilles H, Heuman DM. Predictors of the recurrence of hepatic encephalopathy in lactulose- treated patients. Aliment Pharmacol Ther. 2010; 31(9): 1012-17. 
17. Sharma P, Sharma BC, Sarin SK. Predictors of nonresponse to lactulose for minimal hepatic encephalopathy in patients with cirrhosis. Liver Int. 2009; 29(9): 1365-71.

18. Pangman VC, Sloan J, Guse L. An examination of psychometric properties of the mini-mental state examination and the standardized minimental state examination: implication for clinical practice. Appl Nurs Res. 2000; 13(4): 209-13.

19. Schomerus H, Hamster W. Neuropsychological aspects of portalsystemicencephalopathy. Metab Brain Dis. 1998; 13: 361-77.

20. Conn HO. Trail making and number connection tests in the assessment of mental state in portal systemic encephalopathy. Dig Dis. 1977; 22(6): 541-50.

21. Mullen KD. Pathogenesis, clinical manifestation and diagnosis of hepatic encephalopathy, Semin in Liver Dis. 2007; 27: 10-17.

22. Gomez EV, Gonzalez AT, Bertot LC, Garcia AY, Rodriguez YS, Perez YM. Arterial blood pressure is closely related to ascites development in compensated HCV related cirrhosis. Plos One. 2014; 9(4): e95736.

23. Angeli $P$, Merkel C. Pathogenesis and management of hepatorenal syndrome in patients with cirrhosis. J Hepatol. 2008; 48: S93-103.

24. Arroyo V, Fernandez J, Ginès P. Pathogenesis and treatment of hepatorenal syndrome. Semin Liver Dis. 2008; 28: 81-95.

25. Angeli $\mathrm{P}$, Wong $\mathrm{F}$, Watson $\mathrm{H}$, Ginès $\mathrm{P}$. Hyponatremia in cirrhosis: results of a patient population survey. Hepatology. 2006; 44(6): $1535-42$.

26. Gaduputi V, Chandrala C, Abbas N, Tariq H, Chilimuri S, Balar B. Prognostic significance of hypokalemia in hepatic encephalopathy. Hepatogastroenterology. 2014; 61(133): 1170-4.

27. Dbouk N, McGuire B. Hepatic encephalopathy: A review ofits pathophysiology and treatment. Curr Treat Options Gastroenterol. 2006; 9: 464-74.

28. Albrecht J, Sonnewald U, Waagepertersen H, Schousboe A.Glutamine in the central nervous system: function anddysfunction. Front Biosci. 2007; 12: 332-43.

29. Shawcross D, OldeDamink S, Butterworth RF, Jalan R.Ammonia and hepatic encephalopathy: the more thingschange, the more they remain the same. Metab Brain Dis. 2005; 20: 169-79.

30. Jover R, Rodrigo R, Felipo V,Insausti R, Sáez-Valeri J, García-Ayllón MS, et al. Brain edema andinflammatory activation in bile duct ligated rats withdiet-induced hyperammonemia: A model of hepaticencephalopathy in cirrhosis. Hepatology. 2006; 43: 1257-66.

31. Cordoba J, Blei AT. Brain edema and hepatic encephalopathy. Semin Liver Dis. 1996; 16: 271-80.

32. Kundra A, Jain A, Banga A, et al. Evaluation of plasma ammonia levels in patients with acute liver failure andchronic liver disease and its correlation with the severity ofhepatic encephalopathy and clinical features of raisedintracranial pressure. ClinBiochem. 2005; 38: 696-9.

33. Qureshi MO, Shafqat F, Salih M, Khokhar N. Factors associated with nonresponse to lactulose in patients with cirrhosis and hepatic encephalopathy. Rawal Med J. 2014; 39(2): 115-118. 